\title{
DETERMINAÇÃO DOS COMPOSTOS FENÓLICOS TOTAIS EM CASCAS DE FRUTAS ENCONTRADAS NO CERRADO BRASILEIRO
}

Determination of total phenolic compounds in fruit shells found in brazilian cerrado

Determinación de total de compuestos fenólicos en conchas de frutas encontradas en cerrado brasileño

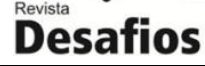

Artigo Original

Original Article

Artículo Original

Rômulo Alves Morais*1,2, André Leonardo dos Santos ${ }^{2}$, Hermanny Matos Silva Sousa ${ }^{1,2}$, Camila Mariane da Silva Soares ${ }^{1,2}$, Diana Lopes da Silva ${ }^{1,3}$, Glêndara Aparecida de Souza Martins ${ }^{1,2}$

${ }^{1}$ Laboratório de Cinética e Modelagem de Processos (LaCiMP) - Universidade Federal do Tocantins

${ }^{2}$ Programa de Pós-Graduação em Ciência e Tecnologia de Alimentos (PPGCTA -UFT) - Universidade Federal do Tocantins

${ }^{3}$ Programa de Pós-Graduação em Biodiversidade e Biotecnologia (REDE BIONORTE) - Universidade Federal do Tocantins

*Correspondência: Laboratório de Cinética e Modelagem de Processos, Escola de Engenharia de Alimentos, Universidade Federal do Tocantins, Quadra 109 Norte, Avenida NS 15, ALCNO-14, Plano Diretor Norte, Palmas TO,CEP: 77001-090.e-mail: romuloitallo2505@gmail.com

Artigo recebido em 20/02/2020 aprovado em 23/03/2020 publicado em 26/03/2020.

\section{RESUMO}

O aumento da produção do setor agroindustrial, ao mesmo tempo em que proporciona benefícios econômicos e nutricionais ao homem, gera uma vasta quantidade de resíduos. Esses resíduos comumente descartados como casca, semente e talos contém uma grande quantidade de compostos bioativos como os compostos fenólicos. Nesse sentido o objetivo desse trabalho foi avaliar a eficácia de três soluções extratoras (água, etanol $80 \%$ e metanol $80 \%$ ) e quantificar o teor de fenólicos totais em sete cascas de espécies de frutas exóticas brasileiras, guapeva (Pouteria gardneriana), jambolão (Syzygium cumini), pitaya (Hylocereus costaricensis), pupunha (Bactris gasipaes), oiti (Licania tomentosa), buriti (Mauritia flexuosa) e pitomba (Talisia esculenta), utilizando o método de FolinCiocalteou. A água mostrou-se a melhor solução extratora para as cascas analisadas. Os teores de compostos fenólicos variaram de 195,27 a 6,59 mg de EAG. $100 \mathrm{~g}^{-1}$ de casca para o extrato aquoso, de 155 a 47,67 mg de EAG. $100 \mathrm{~g}^{-1} \mathrm{de}$ casca para as soluções etanólicas e de 192,57 a 1,35 mg de EAG. $100 \mathrm{~g}^{-1}$ de casca para as soluções metanólicas. Sendo que os melhores resultados foram atribuídos as cascas de pitomba, buriti e guapeva. As cascas avaliadas neste estudo, podem ser consideradas boas fontes de compostos fenólicos totais.

Palavras-chave: Folin-Ciocalteou, resíduos, compostos bioativos.

\section{ABSTRACT}

Increasing production in the agro-industrial sector, in addition to providing economic and nutritional benefits to man, generates a large amount of waste. These generally discarded wastes such as bark, seed and stem contain a large amount of bioactive compounds, such as phenolic compounds. In this sense, the objective of this work was to evaluate the effectiveness of three extraction solutions (water, 80\% ethanol and 80\% methanol) and to quantify the total phenolic content in seven bark of Brazilian exotic fruit species, guapeva (Pouteria gardneriana)., jambolan (Syzygium cumini), pitaya (Hylocereus costaricensis), peach (Bactris gasipaes), oiti (Licania tomentosa), buriti (Mauritia flexuosa) and pitomba (Talisia esculenta) using the Folin-Ciocalteou method. Water proved to be the best extraction solution for the peels analyzed. The content of phenolic compounds ranged from 195.27 to $6.59 \mathrm{mg}$ EAG. 100g-1 bark for the aqueous extract, from 155 to $47.67 \mathrm{mg}$ EAG. 100g-1 bark for ethanolic solutions and 192.57 to 
1.35 mg EAG. 100g-1 housing for methanolic solutions. The best results were attributed to pitomba, buriti and guapeva barks. The peels evaluated in this study can be considered good sources of total phenolic compounds.

Keywords: Folin-Ciocalteou, waste, bioactive compounds.

\section{RESUMEN}

El aumento de la producción en el sector de agronegocios, además de proporcionar beneficios económicos y nutricionales para los humanos, genera una gran cantidad de desechos. Estos desechos generalmente desechados, como la cáscara, las semillas y los tallos, contienen una gran cantidad de compuestos bioactivos, como los compuestos fenólicos. En este sentido, el objetivo de este trabajo fue evaluar la efectividad de tres soluciones de extracción (agua, 80\% de etanol y 80\% de metanol) y cuantificar el contenido fenólico total en siete cáscara de especies exóticas de frutas brasileñas, guapeva (Pouteria gardneriana), jambolan (Syzygium cumini), pitaya (Hylocereus costaricensis), pupuha (Bactris gasipaes), oiti (Licania tomentosa), buriti (Mauritia flexuosa) y pitomba (Talisia esculenta) utilizando el método Folin-Ciocalteou. El água demostró ser la mejor solución de extracción para las exfoliaciones analizadas. El contenido de compuesto fenólico varió de 195.27 a $6.59 \mathrm{mg}$ de EAG. $100 \mathrm{~g}-1$ de cáscara para el extracto acuoso, de 155 a 47,67 mg de EAG. $100 \mathrm{~g}-1$ de cáscara para soluciones de etanol y 192.57 a 1.35 mg de EAG. 100g-1 para soluciones metanólicas. Los mejores resultados se atribuyeron a pitomba, buriti y corteza de guapeva. Las exfoliaciones evaluadas en este estudio pueden considerarse buenas fuentes de compuestos fenólicos totales.

Descriptores: Folin-Ciocalteou, residuos, compuestos bioactivos.

\section{INTRODUÇÃO}

As frutas são cada vez mais indispensáveis na nutrição humana devido à sua composição nutricional, tendo benefícios eficazes a saúde (ALBUQUERQUE et al., 2016), e um dos grandes desafios é produzir alimentos para uma população em crescimento constante, sendo o Brasil um dos principais produtores de frutas do mundo com mais de 150 milhões de hectares biodisponíveis (COUTO, 2015).

Diante disso o bioma Cerrado é caracterizado pela grande diversidade de sua flora, a qual abrange tanto campos abertos até densas florestas, sua flora representa em torno de $30 \%$ das riquezas naturais do Brasil. O Cerrado ocupa $21 \%$ do território nacional e apresenta clima quente, semiúmido e sazonal, e muitas espécies se destaca pelo grande potencial econômico, devido as características peculiares, formas, cores, aromas e sabores variados. Embora tendo grandes variedades, as frutas do cerrado são pouco conhecidas fora da região ao qual são coletadas (GONDIM et al., 2013).
Algumas espécies frutíferas do Cerrado são reconhecidas por possuírem altos valores nutritivos, sendo superior a valores encontrados em espécies cultivadas tradicionalmente e de grande comercialização, nesse contexto podemos destacar alguns frutos com o buriti (Mauritia flexuosa L. f.) uma das fontes vegetais mais ricas em provitamina A, tendo seu valor superior ao da cenoura, ou a cagaita (Stenocalyx dysentericus) rica em proteínas e fonte de minerais dentre outros (PEREIRA \& SANTOS, 2015; CARNEIRO, 2016).

Nesse sentido vários estudos reportam que as frutas nativas, além de nutrir, contém substâncias que podem propiciar benefícios adicionais à saúde, sendo tais benefícios atribuídos à presença de compostos bioativos como os compostos fenólicos, dos quais muitos com ação antioxidante, eficazes na proteção contra doenças crônicas não transmissíveis, tais como doenças cardiovasculares e câncer (ALU'DATT et al.; 2017; CELANT et al., 2015; GHAGANE et al., 2017; PINTAĆ et al., 2018). Entretanto, quando em concentração muito elevada ou em composição 
inadequada, estes compostos podem conferir características indesejáveis, como o escurecimento enzimático de frutas e a interação com proteínas, carboidratos e minerais (IMEH; KHOKHAR 2002).

Por sua vez os compostos fenólicos são definidos como substâncias que possuem um anel aromático com um ou mais substituintes hidroxílicos, incluindo seus grupos funcionais. Estão amplamente distribuídos no reino vegetal, englobando desde moléculas simples até outras com alto grau de polimerização (SOARES et al., 2008).

Contudo apesar do constante crescimento do setor de fruticultura, existe uma grande preocupação com os resíduos gerados, onde são descartados de forma inadequada, gerando grandes volumes, e isso representa um grande problema de contaminação ambiental, principalmente nos recursos hídricos e solo, além disso, cria um ambiente propício para a proliferação de vetores transmissores de doenças, que causam risco à saúde humana (ARAGÃO, 2010).

Desse modo, este trabalho teve como objetivo avaliar a eficiência do solvente extrator e quantificar os teores de compostos fenólicos totais, empregando, respectivamente, o método de Folin-Ciocalteau em diferentes cascas de frutos não convencionais.

\section{MATERIAIS E MÉTODOS}

\section{Seleção dos frutos}

Os frutos in natura utilizados paras as análises foram coletados entre os meses de julho a outubro de 2019, sendo colhidas sete espécies distintas: guapeva (Pouteria gardneriana Radlk), jambolão (Syzygium cumini), pitaya (Hylocereus costaricensis), pupunha (Bactris gasipaes), oiti (Licania tomentosa), buriti (Mauritia flexuosa), pitomba (Talisia esculenta). Os frutos foram colhidos no cerrado local da cidade de Palmas, capital do Estado do Tocantins, sendo os frutos selecionados de acordo com o estado de maturação e conservação, sendo coletados apenas os frutos que apresentassem uma uniformidade visível. Os frutos foram, então, encaminhados para o laboratório de Cinética e Modelagem de Processos da Universidade Federal do Tocantins, onde foram devidamente lavadas em água clorada a 3\%, permanecendo imersas e em repouso durante cerca de 20 minutos para eliminar detritos e possíveis resíduos. As frutas foram novamente lavadas em água corrente para eliminar possíveis sujeiras ainda existentes, em seguida os mesmos foram devidamente embalados e armazenados até o momento do processamento.

\section{Obtenção dos extratos}

Os frutos foram cortados em pequenos pedaços, e separados entre polpa e casca, sendo o intuito do trabalho avaliar apenas a casca. Foram avaliados três solventes distintos para extração a frio de compostos fenólicos nas diferentes amostras de cascas: água (extrato aquoso), etanol a $80 \%$ (extrato etanólico) e metanol 80\% (extrato metanólico), Para cada solvente testado, foram pesados um grama de casca fresca homogeneizada em erlenmeyer de $50 \mathrm{ml}$, aos quais foram adicionados $10 \mathrm{ml}$ da solução extratora, em quadruplicata (OLIVEIRA et al., 2012).

\section{Compostos fenólicos totais}

A determinação dos compostos fenólicos totais, nos extratos etanólicos, metanólicos e aquosos, foi realizada utilizando-se o reagente de FolinCiocalteau, conforme metodologia descrita por Waterhouse (2002), com leitura em espectrofotômetro digital em absorbância de $750 \mathrm{~nm}$. Dos extratos foram retiradas alíquotas de $100 \mu \mathrm{L}$ e adicionado $0,2 \mathrm{~mL}$ do reagente Folin-Ciocalteau e $2 \mathrm{ml}$ de água deionizada, após aguardar três minutos foi adicionado $1 \mathrm{ml}$ de carbonato de sódio a 7,5\% (v/v), em seguida a solução ficou em repouso por duas horas sem contato com a luz, e foi auferida a absorbância. O método se baseia no estabelecimento de uma curva padrão de ácido 
gálico, na faixa de 100 a $500 \mathrm{mg} \mathrm{L}^{-1}$. Onde os resultados foram expressos em $\mathrm{mg}$ de equivalentes de ácido gálico (EAG) por 100 g de amostra.

\section{Análise estatistica}

A análise dos resultados obtidos foi realizada pela aplicação da ANOVA e teste Tukey visando identificar diferenças significativas entre as médias obtidas para cada solvente, utilizando o software Statistic $^{\circledR}$ 7.0. O nível de significância para a diferença entre as médias foi de $5 \%(\mathrm{p}<0,05)$. Todas as análises foram realizadas em quadruplicata e os resultados apresentados como média \pm desvio padrão (FERREIRA, 2014).

\section{RESULTADOS E DISCUSSÃO}

Os compostos fenólicos, presentes nas frutas e hortaliças, são um dos principais responsáveis pela atividade antioxidante destas. Seu conteúdo final pode estar influenciado por fatores como: a maturação, a espécie, práticas de cultivo, origem geográfica, estágio de crescimento, condições de colheita e processo de armazenamento (SOARES, 2008)

Observando os resultados da tabela 01, podemos verificar que a melhor solução extratora para os compostos fenólicos totais foi a água para a maiorias das cascas analisadas, valores esses que ficaram bem próximos dos resultados obtidos para os extratos metanólicos. Vale salientar que a solução extratora álcool $80 \%$ não obteve resultados de relevância quando comparado aos demais.

Os resultados obtidos sobre a composição fenólica total de casca de frutos exóticos brasileiros nos fazem perceber que em grande maioria das cascas analisadas são boas fontes de compostos fenólicos $(195,27$ a 47,67 mg de EAG. $100 \mathrm{~g}^{-1}$ de casca) com exceção da casca da pitaya que obteve valores inferiores as demais cascas reportadas aqui nesse estudo $(6,59$ a $1,35 \mathrm{mg}$ de EAG. $100 \mathrm{~g}^{-1}$ de casca) vale ressaltar que a para a solução etanólica a casca de pitaya não apresentou resultados, não sendo então detectado compostos fenólicos totais pela metodologia proposta por Waterhouse (2002) e Oliveira et al., (2012).

Ao compararmos os resultados aqui obtidos com as cascas de frutas produzidas e consumidas normalmente podemos verificar que as casca desses frutos exóticos se destacam superiormente se avaliarmos os teores de fenólicos totais da goiaba, maçã, romã e uva $(22,01,3,67,36$ e 3,25 $67 \mathrm{mg}$ de EAG. $100 \mathrm{~g}^{-1}$ de casca respectivamente) (BARROS, 2011; KESSIN et al., 2018), casca do maracujá (2,53, 2,30, 2,06 mg de EAG. $100 \mathrm{~g}^{-1}$ de casca para os extratos aquoso, metanólico e etanólico respectivamente) (CAZARIN et al., 2014). Nesse contexto os extratos que contêm maiores conteúdos de fenóis totais em ordem decrescente foram: extratos aquosos e etanólico da casca da guapeva $(195,27$ e 133,45 mg de EAG. $100 \mathrm{~g}^{-1}$ de casca), extrato metanólico da casca de pitomba (192, 57 mg de EAG. $100 \mathrm{~g}^{-1}$ de casca), extrato metanólico da casca do buriti $(159,29 \mathrm{mg}$ de EAG. $100 \mathrm{~g}^{-1}$ de casca) tais valores foram superiores a cascas de frutos também considerados exóticos como a cagaita (18,38 mg de EAG. $100 \mathrm{~g}^{-1}$ de casca), araticum (40,7 mg de EAG. $100 \mathrm{~g}^{-1}$ de casca) e lobeira $(35,15 \mathrm{mg}$ de EAG. $100 \mathrm{~g}^{-1}$ de casca) (ROESLER et al., 2007). O aproveitamento total dos compostos fenólicos desses extratos deverá ser estudado por meio de estudos adicionais dos parâmetros empregados no processo de extração como razão solvente, massa, tempo de extração, afim de verificar as melhores condições de extração dos compostos fenólicos totais presentes em casca de frutos não convencionais. 
Tabela 01 - Teor de compostos fenólicos totais e avaliação da melhor solução extratora para casca de frutos exóticos brasileiros.

\begin{tabular}{cccc}
\hline CASCA DE FRUTAS & \multicolumn{2}{c}{ COMPOSTOS FENÓLICOS TOTAIS (mg EAG/100 g de amostra) } \\
\cline { 2 - 4 } ÁGUA & ETANOL 80\% & METANOL 80\% \\
$\begin{array}{c}\text { GUAPEVA } \\
\text { Pouteria gardneriana } \text { Radlk } \\
\text { JAMBOLÃO }\end{array}$ & $195,27 \pm 0,96^{\mathrm{aA}}$ & $133,45 \pm 0,52^{\mathrm{bB}}$ & $113,34 \pm 0,44^{\mathrm{cC}}$ \\
$\begin{array}{c}\text { Syzygium cumini } \\
\text { PITAYA }\end{array}$ & $105,74 \pm 0,68^{\mathrm{cC}}$ & $115,20 \pm 0,74^{\mathrm{bB}}$ & $121,28 \pm 0,46^{\mathrm{aC}}$ \\
$\begin{array}{c}\text { Hylocereus costaricensis } \\
\text { PUPUNHA }\end{array}$ & $6,59 \pm 0,63^{\mathrm{aE}}$ & $\mathrm{ND}$ & $1,35 \pm 0,44^{\mathrm{bF}}$ \\
$\begin{array}{c}\text { Bactris gasipaes } \\
\text { OITI }\end{array}$ & $80,74 \pm 0,88^{\mathrm{aD}}$ & $47,67 \pm 0,20^{\mathrm{bD}}$ & $48,65 \pm 0,44^{\mathrm{bE}}$ \\
$\begin{array}{c}\text { Licania tomentosa } \\
\text { BURITI }\end{array}$ & $84,80 \pm 0,74^{\mathrm{aD}}$ & $72,80 \pm 0,36^{\mathrm{bC}}$ & $72,97 \pm 0,44^{\mathrm{bD}}$ \\
$\begin{array}{c}\text { Mauritia flexuosa } \\
\text { PITOMBA }\end{array}$ & $143,92 \pm 0,73^{\mathrm{cB}}$ & $155,07 \pm 0,83^{\mathrm{bA}}$ & $159,29 \pm 0,93^{\mathrm{aB}}$ \\
Talisia esculenta & $162,33 \pm 0,69^{\mathrm{bB}}$ & $143,58 \pm 0,58^{\mathrm{cA}}$ & $192,57 \pm 1,05^{\mathrm{aA}}$ \\
\hline
\end{tabular}

Médias seguidas de mesma letra minúscula na linha e de mesma letra maiúscula na coluna não apresentam diferença significativa pelo Teste de Tukey ao nível de $5 \%$ de probabilidade $(\mathrm{p}<0,050)$.

Ao avaliarmos a tabela 01 em relação as soluções extratoras testadas podemos verificar que apenas o oiti (Licania tomentosa) e a pupunha (Bactris gasipaes) não diferiram estatisticamente a $5 \%$ para os extratos etanólico e metanólico diferindo apenas para o extrato aquoso, as demais cascas das frutas exóticas guapeva, jambolão, pitaya, buriti e pitomba ambas diferiram estatisticamente a $5 \%$ de probabilidade em relação as soluções extratoras.

Tabela 02 - Análise de variância do desdobramento casca de fruta em relação aos solventes testados.

\begin{tabular}{cccccc}
\hline FV & GL & SQ & QM & Fc & Pr $>$ Fc \\
\hline Casca_fruta & 6 & 383279.634921 & 63879.939153 & 146877.232 & 0.0000 \\
Solvente & 2 & 5370.015873 & 2685.007937 & 6173.558 & 0.0000 \\
Casca_fruta*solvente & 12 & 30303.317460 & 2525.276455 & 5806.293 & 0.0000 \\
Erro & 105 & 45.666667 & 0.434921 & & \\
\hline Total corrigido & 125 & & & \\
CV $(\%)$ & 0,64 & Número de observações: & & \\
Média geral: & 102,6507 & \multicolumn{3}{c}{126} \\
\hline
\end{tabular}

Analisando separadamente cada solução extratora em relação as cascas em estudo, verificamos que para o extrato aquoso, buriti e pitomba não difeririam entre si, o mesmo foi constatado para a pupunha e o oiti. Ao analisarmos os extratos etanólicos verificamos que a pitomba e o buriti novamente não se diferiram entre si, assim como a guapeva e jambolão. Já para o extrato metanólico apenas guapeva e jambolão não diferiram entre si a 5\% de probabilidade pelo teste de Tukey. 
Tabela 02 - Resumo das análises de variância das soluções extratoras água, etanol 80\% e metanol

$80 \%$ de sete cascas de frutos exóticos.

\begin{tabular}{cclll}
\hline FV & GL & & \multicolumn{2}{c}{ QM } \\
\hline & & Água & Etanol 80\% & Metanol 80\% \\
\cline { 3 - 5 } Casca_fruta & 6 & 23407.373016 & 19523.039683 & 26000.079365 \\
Solvente & 0 & 0.0000000 & 0.0000000 & 0.0000000 \\
Casca_fruta*solvente & -6 & 23407.373016 & 19523.039683 & 26000.079365 \\
\hline Cv (\%) & & 52,63 & 56,04 & 60,86 \\
Média geral: & & 111.2142857 & 95.3809524 & 101.3571429 \\
\hline \multicolumn{4}{c}{ Número de observações: 42 } \\
\hline
\end{tabular}

Ao fazermos uma análise mais abrangente sobre os solventes utilizados (tabela 01 e 02 ), verificamos que para as cascas analisadas os melhores resultados foram atribuídos ao extrato aquoso, isso se deve que a maior parte dos compostos fenólicos dessas cascas apresentam maior polaridade, portanto são mais hidrossolúveis. Tais resultados são corroborados por $\mathrm{Wu}$ et al., (2004) e Vieira et al. (2011), que ao avaliarem o conteúdo de polifenóis de frutas consumidas nos Estados Unidos e Brasil respectivamente, observaram que a fração hidrofílica possuía uma quantidade bem superior desses constituintes que a porção lipofílica.

Figura 01 - Média das absorbâncias obtidas para os diferentes extratores em sete variedades de cascas de frutos exóticos cultivados no Brasil.

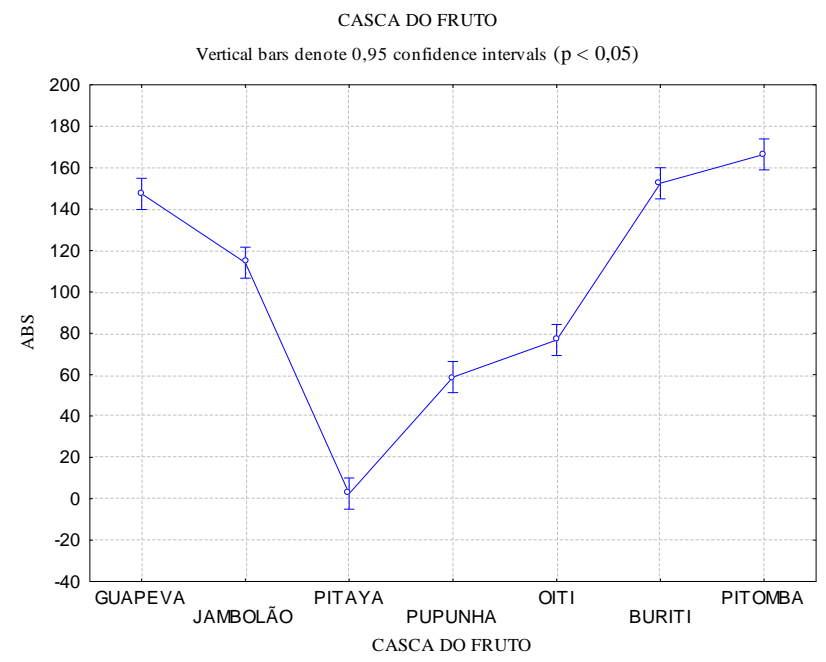

Analisando a figura 01 verificamos que a casca que teve melhor média de extração dos fenólicos totais foi a pitomba seguida de buriti e guapeva.

\section{CONCLUSÃO}

Os resultados obtidos no presente estudo demonstram que as cascas dos frutos exóticos, subproduto de descarte, poderia ser utilizada como fonte de compostos fenólicos, sendo as cascas de pitomba, buriti e guapeva as mais promissoras. Sua incorporação na formulação de alimentos deve atentar as suas características físicas, químicas e sensoriais, para garantir seu melhor aproveitamento e aceitação entre os consumidores.

\section{AGRADECIMENTOS}

A toda equipe do Laboratório de Cinética e Modelagem de Processos, ao Laboratório de Pesquisa Agropecuária, ao $\mathrm{CNPq}$ pelo apoio financeiro para o desenvolvimento dessa pesquisa.

Todos os autores declararam não haver qualquer potencial conflito de interesses referente a este artigo.

\section{REFERÊNCIAS}

ALBUQUERQUE, T. G.; SANTOS, F.; SANCHESSILVA, A.; OLIVEIRA, M. B.; BENTO, A. C.; Nutritional and phytochemical composition of Annona cherimola Mill. fruits and by-products: Potential health benefits. Food chemistry, 193, 187-195, 2016.

ALU'DATT, M.H. A review of phenolic compounds in oil-bearing plants: Distribution, identification and 
occurrence of phenolic compounds. Food Chemistry, 218, 99-106, 2017.

ARAGÃO, A. S. L. Utilização de coprodutos da fruticultura do Vale do São Francisco na alimentação de ruminantes. Dissertação (Mestrado em Ciência Animal). Universidade Federal do Vale do São Francisco. Petrolina, PE. 2010.

BARROS, Z. M. P. Cascas de frutas tropicais como fonte de antioxidantes para enriquecimento de suco pronto. Tese de Doutorado. Universidade de São Paulo. 2011.

CARNEIRO, N. S. Caracterização química e avaliação da atividade antioxidante da polpa e óleo essencial da pera do cerrado (Eugenia klotzschiana Berg.). Dissertação (Mestrado em Agroquímica). Instituto Federal Goiano - Campus de Rio Verde. Rio Verde, GO. 2016.

CAZARIN, C. B. B.; DA SILVA, J. K.; COLOMEU, T. C.; DE LIMA ZOLLNER, R.; JUNIOR, M. R. M. Capacidade antioxidante e composição química da casca de maracujá (Passiflora edulis). Ciência Rural, 44(9), 1699-1704. 2014.

CELANT, A.; MAGRI, D.; STASOLLA, F. R. Collection of plant remains from archaeological contexts. In: Plant Microtechniques and Protocols. Springer, Cham, p. 469-485, 2015.

COSTA, H. S. Nutritional and phytochemical composition of Annona cherimola Mill. fruits and byproducts: Potential health benefits. Food chemistry, v. 193, p. 187-195, 2016.

COUTO, M. L. B. G. Estudo do processo de secagem de frutos do cerrado em secador de bandejas com circulação forçada de ar. Trabalho de conclusão de curso (Bacharelado em Química Tecnológica) Universidade de Brasília, Brasília, DF. 2015.

FERREIRA, D F. Sisvar: a Guide for its Bootstrap procedures in multiple comparisons. Ciência Agrotécnica, v.38, n.2, 2014.

GHAGANE, S. C.; PURANIK, S. I.; KUMBAR, V. M.; NERLI, R. B.; JALALPURE, S. S.; HIREMATH, M. B.; ALADAKATTI, R. In vitro antioxidant and anticancer activity of Leea indica leaf extracts on human prostate cancer cell lines. Integrative medicine research, v. 6, n. 1, p. 79-87, 2017.

GONDIM, P. J.; DE M SILVA, S.; PEREIRA, W. E.; DANTAS, A. L.; CHAVES NETO, J. R.; DOS SANTOS, L. F. Qualidade de frutos de acessos de umbu-cajazeira (Spondiassp.). Revista Brasileira de Engenharia Agrícola e Ambiental, v. 17, n. 11, p. 1217-1221, 2013.
IMEH. U.; KHOKHAR. S. Distribution of Conjugated and Free Phenols in Fruits: Antioxidant Activity and Cultivar Variations. Journal of Agricultural and Food Chemistry, Washington, v. 50, p. 6.301- 6.306, 2002.

KESSIN, J. P. et al. Atividade antioxidante de compostos fenólicos presentes em polpa e casca de goiabeira serrana. Brazilian Journal of Food Research, Campo Mourão, v. 9, n. 1, p. 141-153, jan./mar. 2018.

OLIVEIRA, P. S.; MÜLLER, R. C. S.; DANTAS, K. D. G. F.; ALVES, C. N.; VASCONCELOS, M. A. M. D.; VENTURIERI, G. C. Phenolic acids, flavonoids and antioxidant activity in honey of Melipona fasciculata, M. flavolineata (Apidae, Meliponini) and Apis mellifera (Apidae, Apini) from the Amazon. Química Nova, v. 35, n. 9, p. 1728-1732, 2012.

PEREIRA, A. C.; DOS SANTOS, E. R. Frutas nativas do Tocantins com potencial de aproveitamento econômico. Agri-environmental sciences, v. 1, n. 1, 2015.

PINTAĆ, D.; MAJKIĆ, T.; TOROVIĆ, L.; ORČIĆ, D.; BEARA, I.; SIMIN, N.; LESJAK, M. Solvent selection for efficient extraction of bioactive compounds from grape pomace. Industrial Crops and Products, v. 111, p. 379-390, 2018.

ROCHA, W. S.; LOPES, R. M.; SILVA, D. D.; VIEIRA, R. F.; SILVA, J. D.; AGOSTINI-COSTA, T. D. S. Compostos fenólicos totais e taninos condensados em frutas nativas do cerrado. Revista Brasileira de Fruticultura, v. 33, n. 4, p. 1215-1221, 2011.

ROESLER, R..; MALTA, L. G.; CARRASCO, L. C.; HOLANDA, R. B.; SOUSA, C. A. S.; PASTORE, G. M. Atividade antioxidante de frutas do cerrado. Food Science and Technology, 27(1), 53-60. 2007.

SOARES, M.; WELTER, L.; KUSKOSKI, E. M.; GONZAGA, L.; FETT, R. Compostos Fenólicos e atividade antioxidante da casca de uvas Niágara e Isabel. Revista Brasileira de Fruticultura, Jaboticabal, v, 30, n. 1, p. 59-64, 2008.

SOARES, M.; WELTER, L.; KUSKOSKI, E. M.; GONZAGA, L.; FETT, R. Compostos fenólicos e atividade antioxidante da casca de uvas Niágara e Isabel. Revista Brasileira de Fruticultura, 30(1), 5964. 2008.

VIEIRA, L. M..; SOUSA, M. S. B.; MANCINIFILHO, J.; LIMA, A. D. Fenólicos totais e capacidade antioxidante in vitro de polpas de frutos 
tropicais. Revista Brasileira de Fruticultura, 33(3), 888-897. 2011.

WATERHOUSE, A. L. Determination of total phenolics. Current protocols in food analytical chemistry, v. 6, n. 1, New York: John Wiley \& Sons. 2002. 